\title{
Amphipathic Alpha Helix
}

National Cancer Institute

\section{Source}

National Cancer Institute. Amphipathic Alpha Helix. NCI Thesaurus. Code C14061.

The amphipathic helix motif is characterized by a repeating pattern of polar $(P)$ and nonpolar (N) side-chains that can be summarized as PxNPPNx. These clusters varied in length from 6 to 15 residues. The conserved non-polar positions are marked by a distinct absence of Asp, Asn and Ser, while other polar side chains are sometimes allowed. 